\title{
Replicating Human-Human Physical Interaction
}

\author{
Kyle B. Reed, James Patton, and Michael Peshkin
}

\begin{abstract}
Machines might physically interact with humans more smoothly if we better understood the subtlety of humanhuman physical interaction. We recently reported that two people working cooperatively on a physical task will quickly negotiate an emergent strategy: typically subjects formed a temporal specialization such that one member commands the early parts of motion and the other the late parts [1]. In our current study, we replaced one of the humans with a robot programmed to perform one of the typical human specialized roles. We expected the remaining human to adopt the complementary specialized role. Subjects did believe that they were interacting with another human but did not adopt a specialized behavior as subjects would when physically working with another human; our negative result suggests a very subtle negotiation takes place in human-human physical interaction.
\end{abstract}

\section{INTRODUCTION}

Understanding human-human physical interaction should lead to an increased understanding of how a robot and a human can intuitively and cooperatively work together physically. When two people work together, they are using their collective force and past knowledge to affect each other and their environment. Sebanz et al. [2] state, in a review of joint action, that it may not be possible to fully understand how humans operate by studying people working in isolation. Understanding how two people work together may elucidate how an individual works alone and how a person would intuitively work with another agent, such as a robot. Klingsport et al. [3] suggest that humanrobot communication in a shared task should follow the implicit human-human communication standards. Learning to implement strategies for how two humans communicate haptically could help in creating more intuitive communication between a robot and a human, which is important as robots are becoming more commonplace and interacting with humans more frequently.

\section{RELATED STUdiES}

There have been several studies examining two subjects cooperatively working on one object in a shared virtual environment. Basdogan et al. [4] performed experiments where two people jointly manipulated objects in a virtual world. The interaction consisted of visual and haptic feedback via

Manuscript received September 14th, 2006.

K. B. Reed is with the Laboratory for Intelligent Mechanical Systems, Northwestern University, Evanston, IL, 60208, USA (reedkb@northwestern.edu)

J. Patton is on a joint appointment with Mechanical Engineering at Northwestern and at the Rehabilitation Institute of Chicago, Chicago, IL 60611, USA

M. Peshkin is with the Laboratory for Intelligent Mechanical Systems, Northwestern University, Evanston, IL, 60208, USA (peshkin@northwestern.edu) the virtual environment. Basdogan et al. found that haptic interaction gave the users a better "sense of togetherness." Hubbold [5] examined two subjects carrying a stretcher in a virtual world. The interaction was both visual and haptic via the virtual environment. Hubbold found that haptic interaction helped give the subjects a better perception of the other member and a "sense of sharing."

Glynn and Henning [6] showed that combining the command from two people controlling the same path-following task resulted in faster and more accurate task execution than one person alone. In their experiment, the acceleration of an inertial mass is controlled by summing the force from each partner. There is no force feedback, only visual feedback, so the subjects are not physically interacting. The resulting force on the mass was the average of the dual human input system without haptic interaction. In a second set of experiments, Glynn et al. [7] added force feedback, so the subjects were interacting through a haptic display, which adds a spring to their interaction. They compared four conditions including both force and position control each with and without force feedback. During force control, the added feedback increased errors while performance time was unchanged. Glynn et al.'s only explanation for larger path deviation was that the feedback interacts with the dynamics of a second order system in complex ways. During position control, the added feedback decreased errors and improved performance.

Fly-By-Wire (FBW), a design for airplane (and car) control, eliminates the direct mechanical connection between the pilot and the plane's control surfaces and also between the two pilots. Depending on the configuration and design of the FBW system, the flight sticks have little or no haptic interaction between pilots. In a comparison between direct mechanical connections and non-haptic FBW, Summers et al. [8] found a performance decrement when using nonhaptic FBW. Field and Harris [9] conducted a survey among commercial aircraft pilots, some who have flown planes with a direct connection and some who have flown using nonhaptic FBW. Field and Harris found that communication was lost, which could adversely affect the pilots' awareness of current situations. Many pilots in the study stated that being able to feel the motions and forces of the other pilot was important and useful. Many pilots also stated that they wanted to be able to feel what the autopilot was doing so they could determine if the plane was flying correctly.

Figure 1 summarizes configurations of several of the above studies which investigated the performance of two people cooperatively manipulating a single object. Two people physically cooperating yield the greatest benefit from working with a partner. 


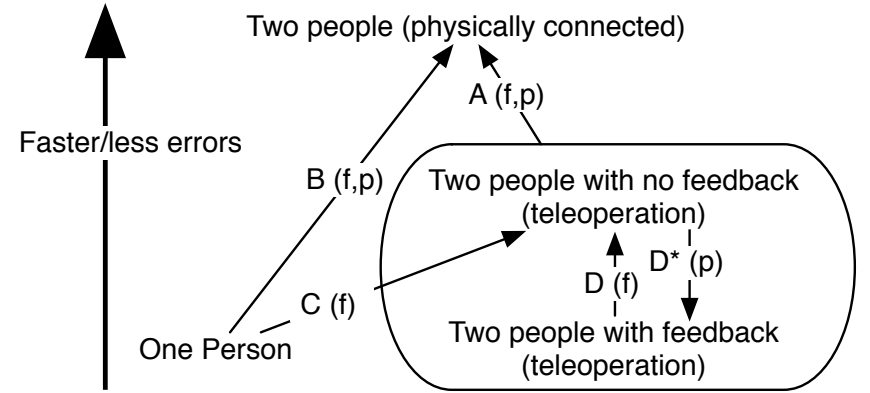

Fig. 1. Summary of various studies comparing the performance of different forms of human-human interactions. All studies involved a real or simulated second order inertial system, except for D*, which was a zeroorder system. Comparisons containing an 'f' indicate that their partner's force was conveyed to their partner while a 'p' indicates position was conveyed in the feedback. \{A-Summers et al. [8] and Field et al. [9]; B-Reed et al. [10]; C-Glynn et al. [6]; D-Glynn et al. [7]\}

In our present research, our goal is to determine what specifically is responsible for the added benefit of a partner and to begin recreating the beneficial interaction in a robotic agent.

Griffin et al. [11], Griffiths and Gillespie [11], and Takubo et al. [12] have demonstrated robots physically working with humans, but their controllers are not based upon the interactions of two people. Their methods predominantly try to stabilize the system while the human manipulates the object.

Rahman et al. [13] investigated two humans physically interacting in a simple 1 DoF placement task. They characterized the humans as either being a master or a follower. The master controls the position of the object; the follower tracks the motion of the master. Rahman et al. [14] worked on modeling the impedance of a human arm by analyzing the resistance an arm will create when it is led through a given path. They found that inertia is constant while damping and stiffness decrease throughout the path. Inoue et al. [15] used the master-follower paradigm and human impedance characteristics with two robots to successfully move an object together.

The three above studies do not discuss haptic communication between the two members and do not take into account how the completion strategy changes from working alone to working with a partner. Our previous work [1] shows that the completion strategy drastically changes such that the subjects specialize: one member becomes the master during the early parts of the motion and the other becomes the master during the late parts. In an attempt to replicate a human partner, our present research will implement the specialization strategy in a robotic partner.

\section{Performance and Specialization}

Our past experiments examined how two subjects cooperated on a physical task without being able to see each other; the subjects could only feel the actions of the other person through the object, a 1-DoF crank. We reported results on the speed of task execution in [10] and results of force specialization in [1], and will summarize only briefly here.
In almost all cases two people working together on the task performed faster than either of the individuals could alone. The faster performance cannot be due to sharing the load since the inertia of the system was doubled in the dyad case. Instead, the subjects each performed different aspects of the task.

We measured the forces applied by each member during the task. The sum of the members' forces ("net force") is the task-relevant force that results in acceleration of the crank. Figure 2 shows the net force, and each member's force for a single trial. The trial shown in the figure is from late in the experiment, after the two subjects adapted to working with each other. The sign convention is that a positive force indicates force toward the target. In the early $(0-180 \mathrm{msec})$ and late $(800-1200 \mathrm{msec})$ parts of the profile, the subjects were pushing against each other, thus with opposite signs. We believe "dyadic-contraction" serves to stabilize the subjects, much like co-contraction does within a single person.

We observe that subjects learned to specialize their force production. Figure 2 shows a typical force production profile for subjects who have learned to specialize. Many early trials did not show specialization while many of the later trials did. In the acceleration part of the profile (180 msec - $420 \mathrm{msec})$, member $\mathrm{A}$ is pushing toward the target (positive force) with enough force so that the crank and member B's arm are pulled along. In the deceleration part of the profile $(420 \mathrm{msec}$ - $750 \mathrm{msec}$ ), member B is decelerating (negative force) with enough force to slow down the crank as well as member A's arm. Thus, member A accelerated the crank while member B decelerated the crank.

The subjects learned to specialize their force production temporally to produce a net force profile similar to a single person, but faster. The subjects arrived at this division of labor solely through a haptic communication channel since no other communication was allowed. Feygin et al. [16] found that haptic guidance helped subjects learn the temporal aspects tasks better than visual guidance did.

Since most of our subjects specialized as described, we hypothesized that a robotic partner that specialized would work well with a human on the same task.

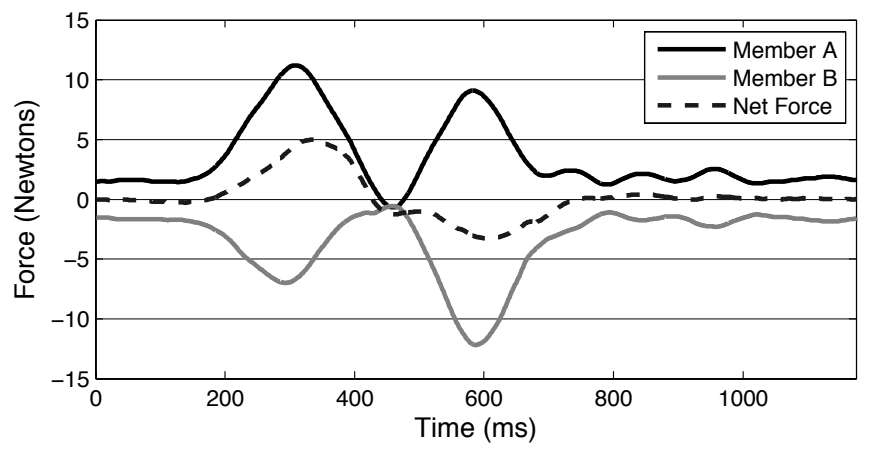

Fig. 2. Force profile of a dyad who developed a specialized completion strategy. Each member only pushes in one direction, yet the forces sum to a force profile similar to an individual performing the task. Each member dominates on a certain aspect of the task: member A accelerates while member B decelerates. 


\section{Simulated PARTNeR}

\section{A. Hypothesis}

Our past research suggests that humans have an ability to cooperate and work together by specializing their forces. The experiment described below was designed to replicate the interaction of two people on a simple task by replacing one person with a simulated partner. The simulated partner is designed to mimic one partner's role in the specialization we found in human-human physical interaction. We expected subjects to work with the robotic partner in the same way as they did with a human partner.

It is possible that subjects who know they are working with a robot will behave differently than if they believe they are working with a human. As a control, some experiments involved a confederate. The confederate stood in as if they were the subject's partner, but the confederate did not work with the subject, the robot did. We hypothesized that a perceived human partner combined with a robotic physical partner would elicit the same response from a subject as working with an actual human partner.

\section{B. Method}

1) Participants: Twenty two students ( 7 men; 1 lefthanded), age 18-24, from Northwestern University's Psychology participant pool participated after giving informed consent.

In eleven of the experiments, a confederate also participated. The confederate pretended to lack knowledge of the experiment. Although the confederate never actually worked with a subject, the subjects would assume they were working with a person. The confederate stood across from the subject during the experiment, but did not physically interact with the subject. The confederate did perform some trials of the task alone to ensure the subjects believed the confederate was participating. In the other eleven experiments, the subjects knowingly worked alone.

2) Apparatus and Stimuli: The device used for our experiments is shown in Figure 3. It is a simple 1 DoF crank with two handles. The handles are connected via a rigid link that can spin freely at the center. A direct drive motor is attached under the table and is unknown to the subject. The angular position of the crank is measured by an optical encoder and displayed to each subject as a bold black mark on the top disk, which turns with the crank. A projector mounted above the table (not shown) displays a target in the same relative position for each subject, a motivating performance measure, and separate instructions to each subject. A curtain hangs between the subjects to prevent visual communication and the subjects are asked not to speak to each other.

The subjects are instructed to move the black mark on the disk into the projected target and hold it there until a new target appears. After a random delay of 700 to $1700 \mathrm{msec}$ with the mark inside the target, a new target appears. Each new target appears on the opposite side of the workspace, so the targets alternate from the left to the right side of the top disk. Each target subtends $6^{\circ}$ of the $50.5 \mathrm{~cm}$ diameter

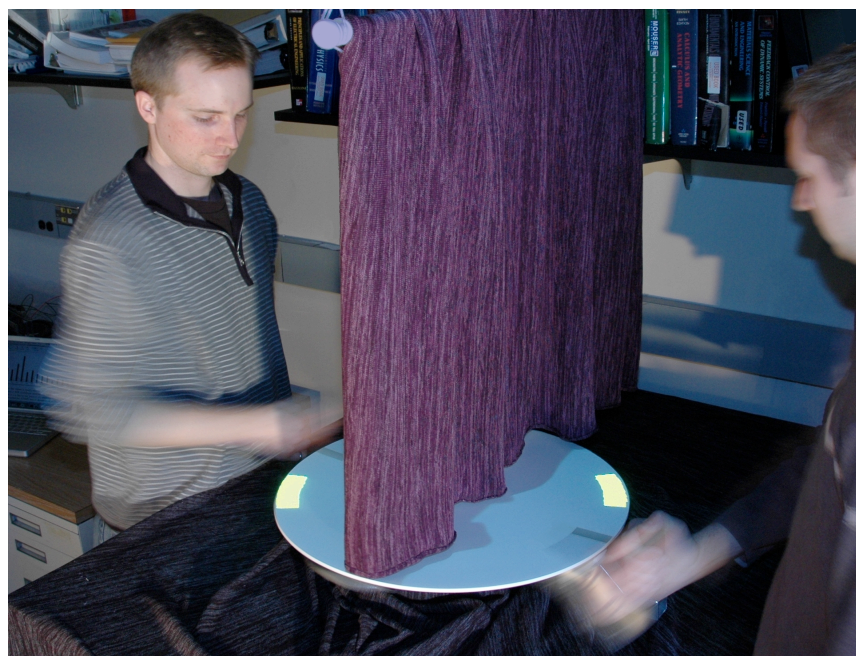

Fig. 3. Experimental setup with two-handled crank. Although shown here with two subjects, the experiment presented here simulated one person with a motor. Half the subjects assumed they were working with the person on the other side while the other half the subjects knew they were not working with a person since no one was there.

disk (2.6 $\mathrm{cm}$ at the perimeter of the disk) with a distance between consecutive targets of $70^{\circ} \pm 10^{\circ}(30.9 \pm 4.4 \mathrm{~cm})$. The targets change color when the crank is inside the target.

3) Robotic Partner: The robotic partner is composed of two parts: an active force production and a simulated inertia. The first part mimics the behavior of a specialized partner who has taken on the role of accelerating the crank. The acceleration part is a modified version of the subject's own force trajectory that was recorded and averaged during the individual trials. When working alone, the subject necessarily did both the acceleration and deceleration parts of the task. We captured the acceleration part of the subject's own force profile, and used it later as the robot's force profile. This acceleration force trajectory was multiplied by 2.1 , which is the typical amount an individual increased his/her force by when he/she becomes part of a dyad [10]. This modified force trajectory becomes a typical force trajectory that could be found in a specialized member of a dyad. We used a recorded version of the subject's forces to account for variations in forces and completion times among subjects, so that any differences can be attributed to the subject working with a copy of themselves and not because the robotic partner is faster than the subject.

The force trajectory for the robotic partner $(R P(t))$ is summarized in equation 1 .

$$
R P(t)=2.1 \times \sum_{i=1}^{100} \frac{f_{i}(t)}{\overline{100}}
$$

where $f_{i}(t)$ is a vector containing the forces for individual trials, $i=1$ to $100, t$ is the time since the target was shown ( $t=1$ to 1300$)$, and $\underline{f_{i}}(t)$ only allows positive values of the force as defined in equation 2 .

$$
\underline{f_{i}}(t)=\left\{\begin{array}{lll}
f_{i}(t) & : & f_{i}(t)>0 \\
0 & : & f_{i}(t) \leq 0
\end{array}\right.
$$


The second part of the robotic partner consists of a simulated inertia. In order to simulate the mass of an arm holding the handle, an inertia similar to that of a human arm was added. Four different confederates assisted throughout the experiments and an average of their arm inertias was used. The inertia $(I)$ of each of their arms was calculated by grabbing the crank with the same grip subjects used in the experiments. The motor applied a torque $(\tau)$ and we measured the angular acceleration $(\alpha)$ over a frequency range from 1 to $35 \mathrm{~Hz}$. The inertia was then found from the equation $I=\frac{\tau}{\alpha}$. The calculated average inertia used was $0.24 \mathrm{~kg} \times \mathrm{m}^{2}$. The simulated force was increased since it would have to accelerate it's own simulated arm as well as the crank. The control loop for the robotic partner as well as measurements of the forces, acceleration and position ran at $1 \mathrm{kHz}$ on a computer running QNX.

4) Procedure: In half the trials we employed a confederate so the subject would think they were working with a person because one might suppose that a subject would cooperate differently if they knew they were working with a person. In this group (Human-Robot-Confederate), an experimental run started with the individual (I) or the confederate (C) performing a block of trials individually. Then the other person completed a block of trials individually. Next, the individual worked with the robotic partner $\left(\mathrm{I}_{\mathrm{m}}\right)$, which the subjects believed to be the other person (i.e. the confederate). This sequence was performed twice, so six subjects performed (I,C,Im,I,C,Im) and five subjects (C,I,Im,C,I,Im). Presentation order made no significant difference.

A confederate was not present in the other half of the experiments. In this group (Human-Robot), an experimental run started with the individual performing a block of trials alone followed by a block of trials in which the individual worked with the robotic partner. The subjects knew there was not a human on the other side of the curtain. This sequence was performed twice, so eleven subjects performed $\left(\mathrm{I}, \mathrm{I}_{\mathrm{m}}, \mathrm{I}, \mathrm{I}_{\mathrm{m}}\right)$.

The experimental apparatus was identical when the subjects were working as individuals and when subjects were working with the robotic partner, except that the small rotational inertia of the crank $\left(0.113 \mathrm{~kg} \times \mathrm{m}^{2}\right)$ was doubled when they were working with the robotic partner. We doubled the inertia since there is twice the available force; both the subject and the robotic partner are applying forces. Also, since the Human-Human studies doubled the inertia in the dyad case, doubling the inertia in the Human-Robot case allows the results to be compared. Each experiment took less than 30 minutes, for a total of 600 trials.

\section{RESUlTS AND Discussion}

\section{A. Change in Performance Time}

The catch trials in the Human-Human experiment were different than the catch trials in the Human-Robot experiment, but the difference in completion times between one person and a dyad (or a person and a person working with the robotic partner) is comparable between experiments. Any difference of having or not having catch trials is eliminated because we are comparing the change in completion time within each experiment.

When two people work together, the dyad performs on average $54.5 \mathrm{~ms}$ faster than the average of the subjects working individually. When a person works with a simulated partner and a confederate is present, the person-robot pair is on average $5.8 \mathrm{~ms}$ faster than the subject is when working alone. There is a significant difference of $48.8 \mathrm{~ms}$ between the Human-Human and Human-Robot completion times $(t(11,15)=3.02, p=0.006)$. When there is not a confederate present, the subject working with the motor is $24.8 \mathrm{~ms}$ slower than working alone, but not a statistically significant amount $(t(11)=1.52, p=0.14)$. The completion time results are summarized in Figure 4.

The eleven subjects without a confederate knew they were working with a non-human agent. Ten of the eleven subjects with a confederate present said they thought they were working with a person. One of them had some doubts, but was not sure either way and this subject's results were not significantly different than the other ten subjects who worked with a confederate. At a conscious level, the subjects working with a confederate believed they were working with a human partner.

When the subjects knew they were not working with a person, the robotic partner hindered the performance relative to working alone. When the subjects believed their partner was human, performance was similar to when the subjects worked alone, which suggests that the origin of forces in physical collaboration can affect the way in which a person will interact with them. The subjects treated a human differently than a robot.

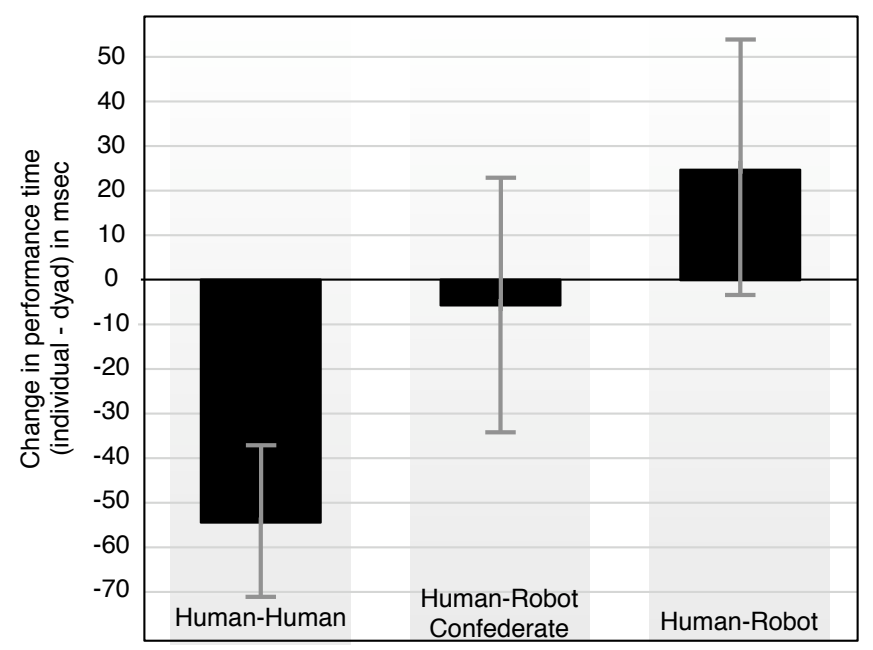

Fig. 4. Comparing the improvement time between the three experimental groups. A negative value means the two people (or human-robot pair) worked faster together than the individuals. Two subjects working together are significantly faster than an individual working with a motor simulating a partner. When working with the robotic partner, subjects with a confederate present are faster than subjects without a confederate present, but not significantly. 
a) Human-Robot

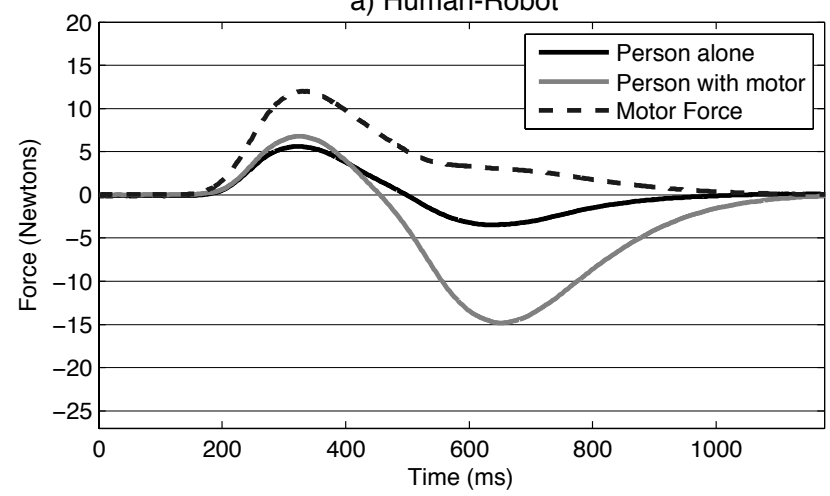

b) Human-Robot-Confederate

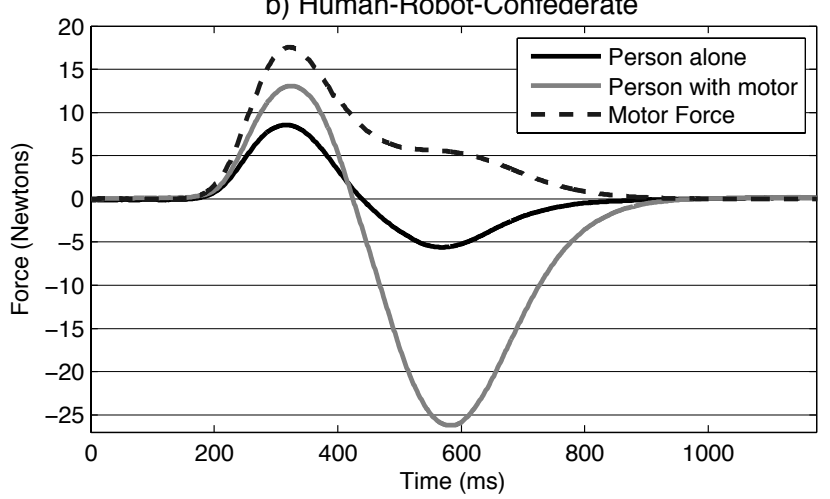

Fig. 5. The average force profiles for individuals working with the motor. All subjects in (a) worked alone and knew they were not working with another human. All subjects in (b) assumed they were working with a confederate, but were working with the robotic partner as in (a). Both graphs show the average profile for the subjects when they worked alone, worked with the motor, and the force from the motor.

It has been suggested that social facilitation could explain why two people are faster than one on the same task. Social facilitation research has a long history [17][18][19] showing that merely having someone present watching a subject causes the subject to perform well on tasks that they are proficient in. One might think that the improved performance of two people working together is due to the second person watching the first, which causes the first person to perform better. Social facilitation could possibly also explain why the Human-Robot-Confederate group was faster than the Human-Robot group. Social facilitation effects could possibly account for part of the performance benefit, but the experimenter was watching the subjects in all cases, so there was always someone visually present, which is the sole requisite for improved performance in most social facilitation studies. Also, social facilitation has only been studied in terms of visual interaction, not physical interaction. During our experiments, the subjects could not see each other due to the curtain and, thus, could only feel each other through the device. Our results suggest that haptic presence could result in a similar performance benefit, which has not been studied in the Psychology literature.

\section{B. Lack of Specialization}

When working with the robotic partner (section IV-B.3), the subjects are given an easy and natural way to specialize. The subject is completely responsible for all the force during deceleration, whereas the subject is free to choose their force during the acceleration phase. The motor applies enough force to accelerate both the crank and the subject's arm. Figure 5 shows the average force of all subjects working alone compared to the same subjects working with the robotic partner. The two force profiles are averaged among all subjects where (a) the confederate is not present and (b) the confederate was present.

None of the subjects developed specialization with the robotic partner. The Human-Robot force profiles shown in Figure 5 convey a very different strategy than two specialized people working together (Figure 2). The Human-Robot force profiles show a strategy that is remarkably similar to an individual performing the task.
We were surprised by the similarity of the subjects' forces when working alone compared to the subjects' forces when working with a robotic partner (Figure 5). During the acceleration phase, the subjects working alone only slightly changed their forces compared to the same subjects working with the robotic partner. The peak force was actually larger in the with-robot case than in the alone case. The larger peak force caused some subjects to apply a negative force earlier in the with-robot case. The robotic partner was designed to accelerate the crank, but the subjects also accelerated the crank, which resulted in too much acceleration force. Thus, the subjects working with the robot had to slow down the crank earlier. The subjects actually worked against the robotic partner and applied a larger overall force than was necessary. As a result, the subjects working with the robotic partner pushed harder during the deceleration phase than in the alone case.

When working alone, each subject knows what the result of their action will be, so each person can accurately predict the outcome of their action. When working with a human partner, the outcome is less predictable since a partner's action is unknown. Sebanz et al. [20] show that a person will internally represent the actions of a person nearby when working on a complementary action. Presumably, haptic interactions also allow two people working together to depend on their partner to complete the complementary action of specialization. We expected that a person would also learn to depend on the robotic partner to complete the complementary action of specialization. Scheidt et al. [21] show that people can adapt to unpredictable forces very quickly. The robotic partner's forces are more predictable than a human's forces. Thus, it is very surprising that the subjects did not learn to work with a predictable robotic partner in the same way as they did when working with an unpredictable human partner. Maybe the unpredictability of a human is beneficial to develop an advantageous cooperation.

Even when the subjects consciously believed they were working with a human partner, the subjects did not specialize their forces. There is evidently a subtle part of human-human physical interaction not replicated by our experiment. 


\section{CONCLUSIONS}

Surprisingly, a robotic partner providing a force profile similar to that which a human partner provides, did not elicit specialization in the human subjects. The robotic partner actually hindered the subject's completion time (relative to working alone) when the subjects knew the force was not a person and had very little effect on completion time when the subjects thought the robotic partner was a person. There must be an aspect of how two people cooperate that is important and missing from our robotic partner. Our negative result suggests a very subtle negotiation takes place in humanhuman physical interaction.

\section{FUTURE WORK}

The research presented here showed that a robot designed to mimic a human parter neither elicited specialization nor improved performance with a human partner. Our future work will attempt to determine what aspects of humanhuman interaction are necessary in order to make a robotic partner that can achieve the same advantages as a human partner.

In our experiments on two subjects working together, we did not interfere with the subjects' interaction. Future experiments could involve blocking certain aspects of their interaction to determine what is important. As an example, we could counteract dyadic-contraction from the humanhuman interaction, which we expect would diminish haptic presence. If shown important, then an improved robotic partner would include dyadic-contraction. Additionally, we would like to test our hypothesize that dyadic-contraction has a stabilizing effect for two humans interacting.

\section{ACKNOWLEDGMENTS}

The authors thank Mitra J. Hartmann, Peter Vishton, Marcia Grabowecky, J. Edward Colgate, Kevin Lynch and Satoru Suzuki for their help with our research. This work was supported by NSF grant ECS-0433948.

\section{REFERENCES}

[1] K. Reed, M. Peshkin, M. J. Hartmann, J. Patton, P. M. Vishton, and M. Grabowecky, "Haptic cooperation between people, and between people and machines," International Conference on Intelligent Robots and Systems (IROS), 2006.

[2] N. Sebanz, H. Bekkering, and G. Knoblich, "Joint action: bodies and minds moving together," TRENDS in Cognitive Sciences, vol. 10, no. 2, pp. 70-76, 2006.
[3] V. Klingsport, J. Demiris, and M. Kaiser, "Human-robot communication and machine learning," Applied Artificial Intelligence Journal, vol. 11, pp. 719-746, 1997.

[4] C. Basdogan, C.-H. Ho, and M. A. Srinivasan, "An experimental study on the role of touch in shared virtual environments," ACM Transactions on Computer-Human Interaction, vol. 7, no. 4, pp. 443-460, 2000.

[5] R. J. Hubbold, "Collaborative stretcher carrying: a case study," Eighth Eurographics Workshop on Virtual Environments, pp. 7-12, 2002.

[6] S. Glynn and R. Henning, "Can teams outperform individuals in a simulated dynamic control task?" Proceedings Of The Human Factors And Ergonomics Society Annual Meeting (ISSN: 10711813), vol. 6, pp. 141-144, 2000.

[7] S. Glynn, R. Fekieta, and R. Henning, "Use of force-feedback joysticks to promote teamwork in virtual teleoperation," Virtual Teleoperation Proc. Of the Human Factors and Ergonomics Society 45th Annual Meeting, 2001.

[8] L. Summers, J. Shannon, T. White, and R. Shiner, "Fly-by-wire sidestick controller evaluation," SAE Technical Paper 871761, Aerospace Technology Conference and Exposition, Long Beach, CA, Society of Automotive Engineers, 1987.

[9] E. Field and D. Harris, "A comparative survey of the utility of cross-cockpit linkages and autoflight systems' backfeed to the control inceptors of commercial aircraft," Ergonomics, vol. 41, no. 10, pp. 1462-1477, 1998.

[10] K. Reed, M. Peshkin, M. J. Hartmann, M. Grabowecky, J. Patton, and P. M. Vishton, "Haptically linked dyads: are two motor control systems better than one?" Psychological Science, vol. 17, no. 5, pp. 365-366, 2006.

[11] W. B. Griffin, W. R. Provancher, and M. R. Cutosky, "Feedback strategies for telemanipulation with shared control of object handling forces," Presence, vol. 14, no. 6, pp. 720-731, 2005.

[12] T. Takubo, H. Arai, Y. Hayashibara, and K. Tanie, "Human-robot cooperative manipulation using a virtual nonholonomic constraint," The International Journal of Robotics Research, vol. 21, pp. 541-553, 2002.

[13] M. Rahman, R. Ikeura, and K. Mizutani, "Cooperation characteristics of two humans in moving an object," Machine Intelligence and Robotic Control, vol. 4, no. 2, pp. 43-48, 2002.

[14] M. Rahman, R. Ikeura, and K. Mitzutani, "Impedance characteristics of human arm for cooperative robot," International Conference on Control, Automation and Systems, pp. 1455-1460, 2002.

[15] Y. Inoue, T. Tohge, and H. Iba, "Object transportation by two humanoid robots using cooperative learning," Evolutionary Computation, 2004. CEC2004. Congress on, vol. 1, pp. 1201-1208, 2004.

[16] D. Feygin, M. Keehner, and F. Tendick, "Haptic guidance: Experimental evaluation of a haptic training method for a perceptual motor skill," Proceedings of the 10th Symposium On Haptic Interfaces For Virtual Environments and Teleoperator Systems (HAPTICS), 2002.

[17] N. Triplett, "The dynamogenic factors in pacemaking and competition," American Journal of Psychology, no. 9, pp. 507-533, 1898.

[18] R. B. Zajonc, "Social facilitation," Science Magazine, vol. 149, pp. 269-274, 1965.

[19] B. H. Schmitt, T. Gilovich, N. Goore, and L. Joseph, "Mere presence and social facilitation: One more time," Journal of Experimental Social Psychology, vol. 22, pp. 242-248, 1986.

[20] N. Sebanz, G. Knoblich, and W. Prinz, "Representing others' actions: just like one's own?" Cognition, vol. 88, pp. 11-21, 2003.

[21] R. A. Scheidt, J. B. Dingwell, and F. A. Mussa-Ivaldi, "Learning to move amid uncertainty," Journal of Neurophysiol, vol. 86, 2001. 